\title{
Biotransformation of ethyl 2-(2'-nitrophenoxy)acetate to benzohydroxamic acid (D-DIBOA) by Escherichia coli
}

\author{
A. Valle ${ }^{\text {a }}$, G. Cabrera ${ }^{\mathrm{a}, *}$, J.M.G. Molinillo ${ }^{\mathrm{b}}$, J.M. Gómez ${ }^{\mathrm{a}}$, F.A. Macías ${ }^{\mathrm{b}}$, D. Cantero $^{\mathrm{a}}$ \\ a Department of Chemical Engineering and Food Technology, University of Cádiz, Avda. República Saharaui s/n, 11510 Puerto Real, Cádiz, Spain \\ b Department of Organic Chemistry, University of Cádiz, Avda. República Saharaui s/n, 11510 Puerto Real, Cádiz, Spain
}

\section{A R T I C L E I N F O}

Article history:

Received 20 May 2010

Received in revised form

15 September 2010

Accepted 15 September 2010

\section{Keywords:}

Biotransformation

Escherichia coli

Serratia marcescens

Benzohydroxamic acid

Natural product

\begin{abstract}
A B S T R A C T
Benzohydroxamic acids, such as DIBOA, exhibit interesting biological properties (herbicidal, fungicidal and bactericidal). Recently, the synthesis of DIBOA has been simplified to only two steps. This paper explores the possibility of replacing the second stage in the chemical synthesis of D-DIBOA by a biotransformation using a strain of Escherichia coli and a strain of Serratia marcescens. Biotransformation experiments were carried out for both strains in the presence of different concentrations $(0.25,0.5$ and $1 \mathrm{mg} / \mathrm{mL}$ ) of the precursor (ethyl 2-(2'-nitrophenoxy)acetate) under aerobic and anaerobic conditions. Both strains tolerated the concentrations of precursor investigated here. Higher biotransformation yields were reached for $E$. coli under aerobic conditions. The UV/vis spectra and ${ }^{1} \mathrm{H} /{ }^{13} \mathrm{C}$ spectroscopic data obtained from HPLC-DAD and NMR, respectively, for the compounds obtained in the biotransformation reaction confirmed the presence of D-DIBOA in cultures of E. coli. The maximum yields were obtained in experiments supplemented with $0.5 \mathrm{mg} / \mathrm{mL}$ of precursor and these were $20.14 \pm 1.87 \%$ under aerobic conditions and $8.17 \pm 0.94 \%$ under anaerobic conditions.
\end{abstract}

(c) 2010 Elsevier Ltd. All rights reserved.

\section{Introduction}

The isolation, structural elucidation, and synthesis of natural products constitute the main source of new molecules with biological activity [1]. Compounds with a (2H)-1,4-benzoxazin$3(4 \mathrm{H})$-one skeleton (benzohydroxamic acids) have attracted the attention of phytochemistry researchers since 2,4 -dihydroxy- $(2 \mathrm{H})$ 1,4-benzoxazin-3(4H)-one (known as D-DIBOA, see Fig. 1) and 2,4dihydroxy-7-methoxy-(2H)-1,4-benzoxacin-3(4H)-one (known as D-DIMBOA, see Fig. 1) were isolated from plants belonging to the Poaceae family $[2,3]$. These compounds exhibit interesting biological profiles that include phytotoxic, antimicrobial, antifeedant, antifungal, and insecticidal properties [4,5]. A number of syntheses of benzohydroxamic acids have been reported in the literature [6-9].

Recently, the synthesis of D-DIBOA has been simplified to only two steps (Fig. 2). The first step involves functionalization of the phenol by nucleophilic substitution to introduce the appropriate side chain (from ethyl bromoacetate) under mild conditions using nitrophenol as the starting material. The reaction product is ethyl 2-(2'-nitrophenoxy)acetate and this is obtained in yields up to $99 \%$.

\footnotetext{
* Corresponding author at: Departamento de Ingeniería Química y Tecnología de Alimentos, Avda. República Saharahui s/n, 11510 Puerto Real, Cádiz, Spain. Tel.: +34 956 016554; fax: +34956016411.

E-mail address: gema.cabrera@uca.es (G. Cabrera).
}

The second step involves reduction of the nitro group followed by a cyclization involving intramolecular addition/removal of the ethyl chain from the ester. This step is heterogeneously catalysed by a $\mathrm{Pd} / \mathrm{C}$ catalyst and the conditions are critical due to the exothermic character of the reaction and the release of hydrogen [6].

One of the alternatives in the total chemical synthesis is the replacement of some stage of the synthesis by a process involving microbial action. This type of process is called biotransformation and can be defined as the modification of a chemical compound by a living organism. There are a wide range of products of secondary origin that can be metabolized and processed by enzymes or microbial cells. These products are interesting in terms of stereoselectivity, regiospecificity and conversion into derivatives, but also for the advantages related to the reaction conditions in which they occur. Such products are currently applied in the production of drugs and other substances of industrial interest.

The hypothesis suggested in this study is that it is possible to reduce the nitro group of ethyl 2-(2'-nitrophenoxy)acetate by a biotransformation that replaces the second stage of the synthesis described above. The biological reduction of nitro groups in a molecule analogous to ethyl 2-(2'-nitrophenoxy)acetate, namely trinitrotoluene (TNT), has been documented in several reports. The reductive transformation of TNT has been documented for many bacterial genera, e.g. Serratia [11], Escherichia [12], Desulfovibrio [13], Clostridium [14,15], and Pseudomonas [16]. Several of these publications indicate that transformation of the nitro group, in aerobic or anaerobic conditions, to an amine occurs through the 
<smiles>O=C1C(O)Oc2ccccc2N1O</smiles>

DIBOA<smiles>COc1ccc2c(c1)OC(O)C(=O)N2O</smiles>

DIMBOA
Fig. 1. Natural benzoxazinones. 2,4-dihydroxy-(2H)-1,4-benzoxazin-3-(4H)-one (DIBOA) and 2,4-dihydroxy-7-methoxy-(2H)-1,4-benzoxazin-3-(4H)-one (DIMBOA) [10].

formation of the corresponding hydroxylamine [12,15-17]. This kind of biotransformation is quite similar to the second stage of the process outlined in Fig. 2. This fact suggested that these microorganisms could be capable of carrying out this step.

The reduction of the nitro groups via hydroxylamines can be attributed to nitroreductases $[18,19]$. In the Enterobacteriaceae family, in addition to the species Escherichia coli, several enzymes with nitroreductase activity have been characterized in Enterobacter cloacae [20], particularly a xenobiotic reductase that reduces nitro groups on the ring to give the hydroxylamine derivative of TNT [21].

The aim of the work described here was to explore the possibility of replacing the second step in the chemical synthesis of D-DIBOA by a biotransformation using a strain of $E$. coli and a strain of Serratia marcescens.

\section{Materials and methods}

\subsection{Microorganisms and chemicals}

The strain E. coli JM109 (ATCC 53323)/pGEM4Z (Promega) was obtained from ATCC. S. marcescens strain N2 belongs to the microbial collection of the Metal Biotechnology Laboratory, Biology Faculty, Havana University. This strain was isolated from a lateritic deposit from Moa, Holguin (Cuba) [22].

The starting compound used to study the biotransformation was ethyl $2-\left(2^{\prime}-\right.$ nitrophenoxy)acetate, which is henceforth referred to as the precursor.

Chemical standards of the precursor and D-DIBOA were synthesized from 2nitrophenol as described by Macías et al. [6]. The reagents were purchased from Sigma-Aldrich and the culture media from Panreac, FLUKA and Cultimed. All organic solvents were HPLC grade.

\subsection{Media}

The inoculums required for experimentation were obtained by seeding bacteria from conservation strains in Luria-Bertani broth (LB). The inoculum/medium ratio was $1: 10(\mathrm{v} / \mathrm{v})$ and the bacteria were grown overnight at $30^{\circ} \mathrm{C}$ in Erlenmeyer flasks. These pre-inoculums were used to inoculate $(10 \%, v / v)$ test systems for both aerobic and anaerobic conditions.

In aerobic experiments $\mathrm{LB}$ broth was inoculated ( $100 \mathrm{~mL}$ final volume) in Erlenmeyer flasks that were kept in an orbital incubator at $150 \mathrm{rpm}$ and $30^{\circ} \mathrm{C}$. For anaerobic cultures LB broth was inoculated ( $50 \mathrm{~mL}$ final volume) in sealed vials and incubation was carried out at $30^{\circ} \mathrm{C}$ under static conditions.

\subsection{Biotransformation experiments}

Strains used in this study were selected for their ability to reduce nitro groups of TNT as this indicates that they may be capable of performing the reduction of the nitro group in the precursor.

The transformation of the precursor was initiated by adding $1 \mathrm{~mL}$ of stock precursor solutions diluted in methanol $(\mathrm{MeOH})$ for the aerobic culture, or $0.5 \mathrm{~mL}$ for the anaerobic culture, to achieve concentrations of $0.25,0.5$ and $1.0 \mathrm{mg}$ of precursor $/ \mathrm{mL}$. The inoculation was performed from cultures of strains $E$. coli and $S$. marcescens in the exponential phase of growth $(10 \%, \mathrm{v} / \mathrm{v})$. During the incubation, $1 \mathrm{~mL}$ and $0.5 \mathrm{~mL}$ samples for aerobic and anaerobic conditions, respectively, were withdrawn at 0 , 9 and $24 \mathrm{~h}$. The samples were harvested by centrifugation for $10 \mathrm{~min}$ at $10,509 \times \mathrm{g}$ and the supernatants were filtered through $0.22 \mu \mathrm{m}$ nylon filters prior to analysis by high-performance liquid chromatography (HPLC).

Two kinds of control experiments were performed: a biotic control, i.e. a bacterial culture without addition of precursor, and several abiotic controls, i.e. culture medium supplemented with the same concentrations of precursor tested without inoculum. The controls were kept under the same conditions of agitation and temperature as for the related experiments.

A biotransformation experiment was subsequently carried out in order to confirm the identities of the reaction products. In this process, E. coli was cultivated in the presence of $0.75 \mathrm{mg} / \mathrm{mL}$ of precursor. The culture was monitored by Thin Layer Chromatography (TLC) during $48 \mathrm{~h}$. After this time, the bacterial culture was centrifuged for $10 \mathrm{~min}$ at $10,509 \times \mathrm{g}$. The supernatant was withdrawn and the pellet was resuspended in distilled water and centrifuged again. The total supernatant from both centrifugations (free of cells) was extracted ten times with ethyl acetate (EtOAc) at room temperature. The solvent was removed from the organic phase on a rotary evaporator under reduced pressure at $35^{\circ} \mathrm{C}$. The resulting crude extract was chromatographed on silica gel columns, using hexane/EtOAc with increasing polarity (from $1: 4$ to $1: 1(\mathrm{v} / \mathrm{v})$ ).

\subsection{Analytical methods}

\subsubsection{Spectrophotometry}

Bacterial growth experiments were monitored by following the increase in turbidity of the culture at $600 \mathrm{~nm}$ using a UV spectrophotometer (Model HITACHI U2001). The bacterial population was represented by counting colony-forming units per $\mathrm{mL}(\mathrm{CFU} / \mathrm{mL})$; this measure was correlated with turbidity by the McFarland scale [23]. All cultures were carried out in duplicate; the values presented are the mean of two experiments.

\subsubsection{High-performance liquid chromatography (HPLC)}

Reverse-phase HPLC analysis was performed on a Merck HITACHI HPLC system equipped with an L-7100 LaChrom quaternary gradient pump, an L-7200 LaChrom autoinjector and an L-7455 LaChrom diode array detector(DAD). Data were collected and processed using a Merck HITACHI D-7000 HPLC data system. Instrumental conditions were Phenomenex ${ }^{\circledR}$ Gemini C18 (4.6 mm $\times 250 \mathrm{~mm}$ ) reversed-phase column at $25^{\circ} \mathrm{C}$. Mobile phases were as follows: water: $1 \% \mathrm{AcOH}(\mathrm{A})$ and $\mathrm{MeOH}: 1 \% \mathrm{AcOH}(\mathrm{B})$ at a flow rate of $1 \mathrm{~mL} / \mathrm{min}$. The injection volume was $15 \mu \mathrm{L}$. The following gradient was used for separation: at $0 \mathrm{~min}, 0 \% \mathrm{~B} ; 7 \mathrm{~min}, 30 \% \mathrm{~B} ; 30 \mathrm{~min}, 40 \% \mathrm{~B} ; 35 \mathrm{~min}, 100 \%$ B.

Chromatogram analysis was carried out using HPLC System Manager software Version 4.1 with the diode array detector set at $253 \mathrm{~nm}$ by comparing the retention times (RT) and ultraviolet/visible spectra with those of the chemical standards.

The fractions eluted from the silica gel column were further purified by HPLC on semipreparative silica gel (Lichrospher Si-60,10 $\mu \mathrm{m}$ ) columns using a mixture of hexane/EtOAc $(6: 4, v / v)$.

\subsubsection{Nuclear magnetic resonance (NMR)}

${ }^{1} \mathrm{H}$ and ${ }^{13} \mathrm{CNMR}$ spectra were run on Varian INOVA-400 spectrometers. Chemical shifts are given in parts per million (ppm) with respect to the residual ${ }^{1} \mathrm{H}$ signal of deuterated chloroform $\left(\mathrm{CDCl}_{3}\right)(\delta 7.25)$ and the ${ }^{13} \mathrm{C}$ signal of chloroform $\left(\mathrm{CHCl}_{3}\right)(\delta$ 77.00).

\subsubsection{Thin layer chromatography (TLC)}

TLC was carried out on Alugram Sil G/UV254 plates (Merck) with a layer thickness of $0.25 \mathrm{~mm}$ with fluorescent indicator. Chromatography plates were developed by treatment with oleum prepared with a solution of sulphuric acid, water and acetic acid $(1: 4: 20)(\mathrm{v} / \mathrm{v})$ followed by heating at $150^{\circ} \mathrm{C}$. The sample spots were observed using a UV lamp at 254 or $360 \mathrm{~nm}$.

\subsection{Biotransformation yield}

For quantitative analysis, stock solutions of each individual chemical standard were prepared by dissolving accurate amounts in LB broth with $1 \% \mathrm{MeOH}(\mathrm{v} / \mathrm{v})$. Working D-DIBOA standard solutions of $0.25,0.125,0.0625,0.0313 \mathrm{mg} / \mathrm{mL}$ were obtained by dilution of the $0.5 \mathrm{mg} / \mathrm{mL}$ stock. These solutions were used to generate the external standard response calibration curves for subsequent measurements of D-DIBOA concentrations from bacteria culture samples. Hydroxamic acids could

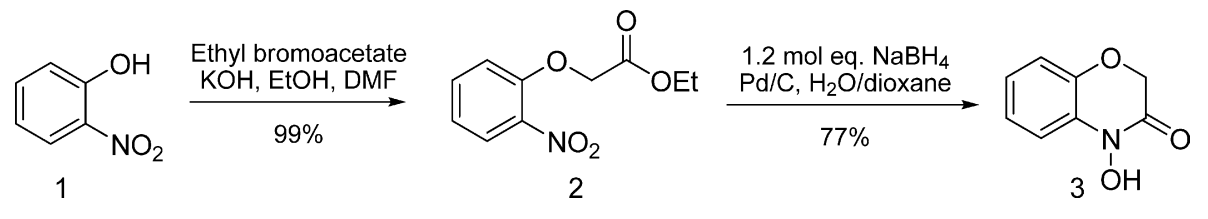

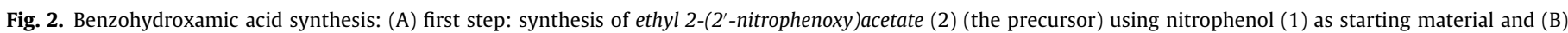
second step: reduction of the precursor nitro group to obtain D-DIBOA (3) [6]. 

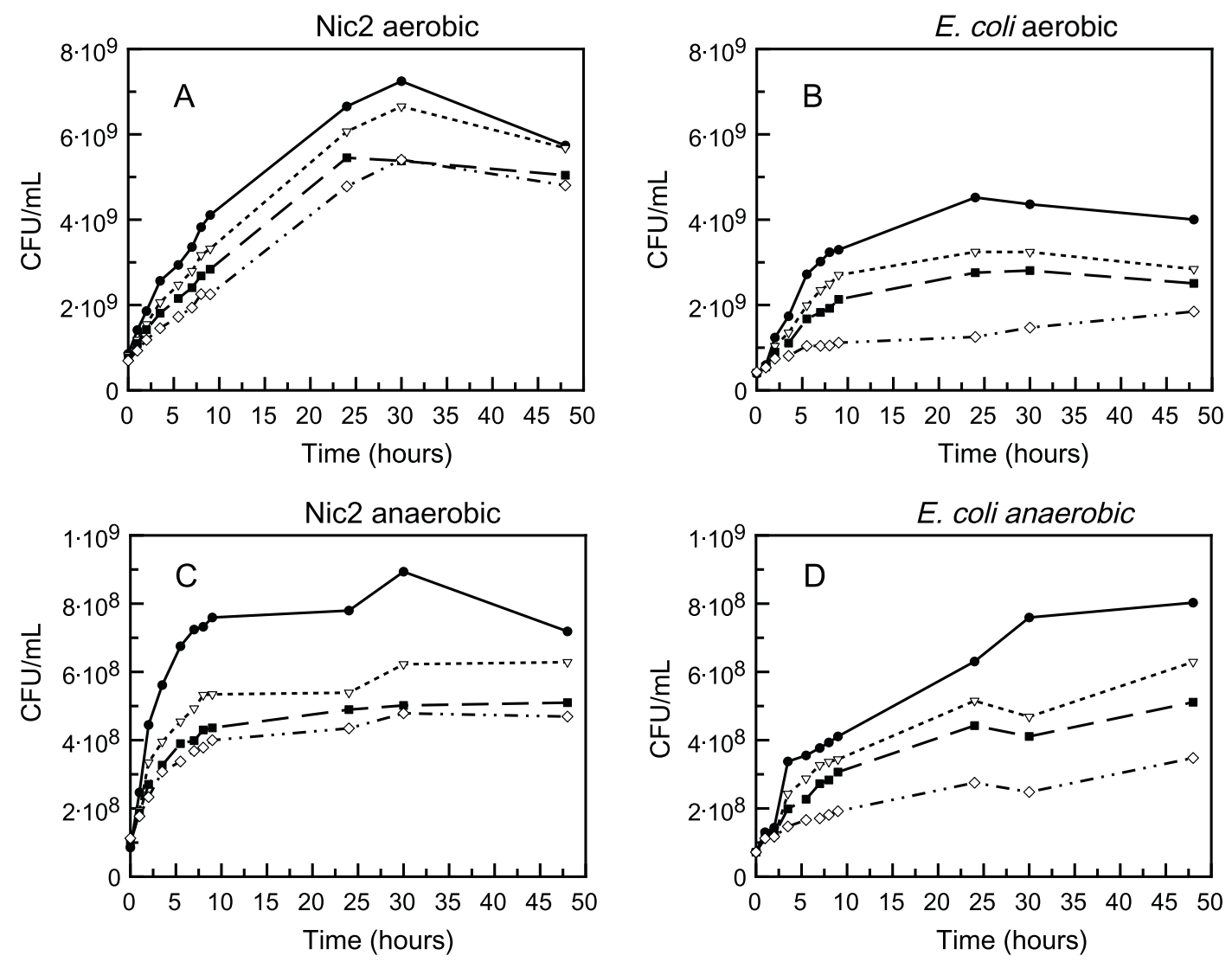

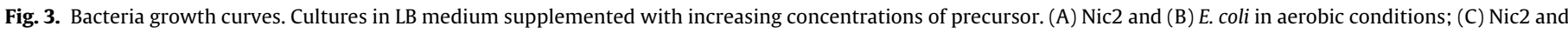

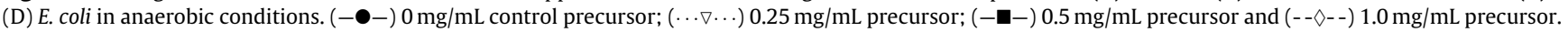

be good chelator of iron, then the quantification of D-DIBOA could be affected if iron complex is formed. For this reason, we determined the influence of the iron (III) concentration present in the medium to D-DIBOA through the examination of $\mathrm{UV} / \mathrm{vis}$ spectra and the peaks areas of the chromatograms (data not shown). Finally, we can conclude that the iron content in LB broth does not affect the quantification of D-DIBOA in the culture conditions.

Data for biotransformation yields are presented as percentage of biotransformation, which was calculated thorough the molar relation of D-DIBOA at $24 \mathrm{~h}$ and the initial quantity of precursor supplemented in each experiment.

\section{Results}

\subsection{Growth curves and response to precursor}

Tracking the growth of $S$. marcescens and E. coli in CFU/mL for $48 \mathrm{~h}$ allowed us to establish the tolerance of these strains to the precursor. Growth was observed in both cultures under aerobic and anaerobic conditions and this was affected by increasing the concentration of precursor. Concentrations above $1 \mathrm{mg} / \mathrm{mL}$ were tested but these proved to be inhibitory for cell growth (data not shown), hence biotransformation experiments were conducted at concentrations below this level.

In general, it appears that the aerobic growth of both strains is similar, with the cell population showing maximum decreases as the concentration of precursor in the medium was increased. However, the absolute $\mathrm{CFU} / \mathrm{mL}$ values achieved are significantly higher for S. marcescens than for E. coli (Fig. 3(A) and (B)). Under anaerobic conditions the behaviour of the two strains was very different, with S. marcescens practically reaching a stationary phase at $10 \mathrm{~h}$ while E. coli peaks are reached at the end of the experiment. In any case, the absolute values for both strains are 10 times smaller than under aerobic conditions.
The different behaviours observed are due to the different natures of the two strains, the different culture conditions tested (aerobic/anaerobic) and the possible inhibitory action exerted by the products that appear in the medium due to microbial metabolism.

\subsection{Identification of compounds by HPLC}

The samples were monitored at $253 \mathrm{~nm}$ and their component/s identified by diode array detector. Three chromatographic peaks were observed in chromatograms of samples from the cultivation of E. coli and S. marcescens at 9 and $24 \mathrm{~h}$ : compound I (Fig. 4(A)) and compound II (Fig 4(B)) appear at RT of 20.41 and $20.74 \pm 0.04 \mathrm{~min}$, respectively, and compound III (Fig. 4(A) and (B)) at $24.69 \pm 0.04 \mathrm{~min}$.

Compounds I and II were identified as D-DIBOA as they eluted very close to the reference standard (RT $20.43 \mathrm{~min}$ ).

In addition, the UV/vis spectra of compounds I and II showed a high correlation to that of the D-DIBOA reference under both sets of conditions, with a correlation coefficient of 0.9999 for compound I and 0.9342 for compound II (Fig. 5(A) and (B), respectively).

The presence of compounds I and II was not evidenced in the chromatograms corresponding to biotic and abiotic controls, thus demonstrating that production of these compounds is the result of the transformation of the precursor by bacteria.

Compound III eluted more slowly than the precursor $(24.51 \pm 0.16 \mathrm{~min})$ and the UV/vis spectrum had a high correlation coefficient with the precursor (0.9962) (Fig. 5(C)). This compound was detected in all of the analyzed samples from both cultures and also the abiotic controls, in both cases with the precursor, but it was not present in the biotic control. These findings seem to indicate that this compound is formed by chemical 

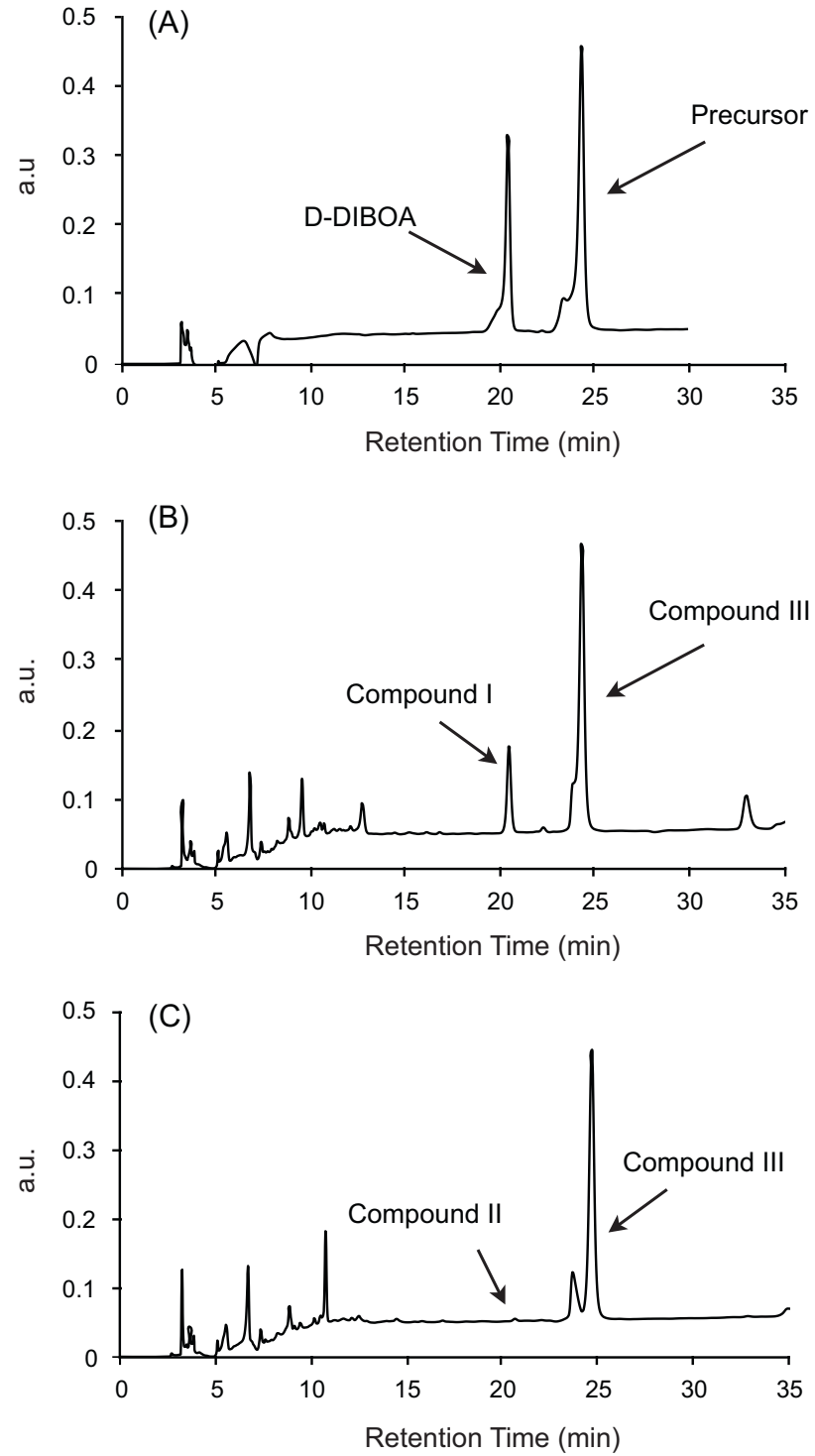

Fig. 4. E. coli and S. marcescens chromatograms. (A) Chromatographic peak areas for reference compounds, D-DIBOA and precursor (B) chromatographic peak areas for compounds in E. coli culture (C) chromatographic peak areas for compounds in $S$. marcescens culture.

degradation of the precursor. However, the structure of compound III remains unknown.

The graphs shown in Fig. 6 represent the chromatographic peak areas obtained for the precursor and compounds I and II under the different conditions tested for E. coli and S. marcescens cultures. It can be seen that the precursor concentration decreases significantly at $9 \mathrm{~h}$ (Fig. 6(A)) and reaches levels below the limit of detection at $24 \mathrm{~h}$ - except for $S$. marcescens cultures under anaerobic conditions, where the concentration is almost constant between 9 and $24 \mathrm{~h}$ (Fig. 6(C)). In the case of E. coli, the decrease in the concentration of precursor during the experiment can be correlated with the increase in the concentration of compound I, as can be seen by the peak areas for this compound in the chromatograms recorded at the two measurement times (Fig. 6(B) and (D)). However, this relationship is not observed in the case of compound II. In this case the disappearance of the precursor cannot be correlated with the appearance of compound II. Moreover, it is evident that $S$. marcescens under anaerobic conditions is not able to transform the precursor, which suggests some inhibitory effect for this compound on the metabolic activity of this strain in the absence of oxygen.

\subsection{Analysis of metabolites in culture supernatants}

Since the experiments with E. coli showed better biotransformation yields of the precursor, it was decided to analyze the metabolites in order to identify and characterize the compounds obtained in these experiments. To this end, a liquid-liquid extraction was carried out on the culture supernatant with ethyl acetate.
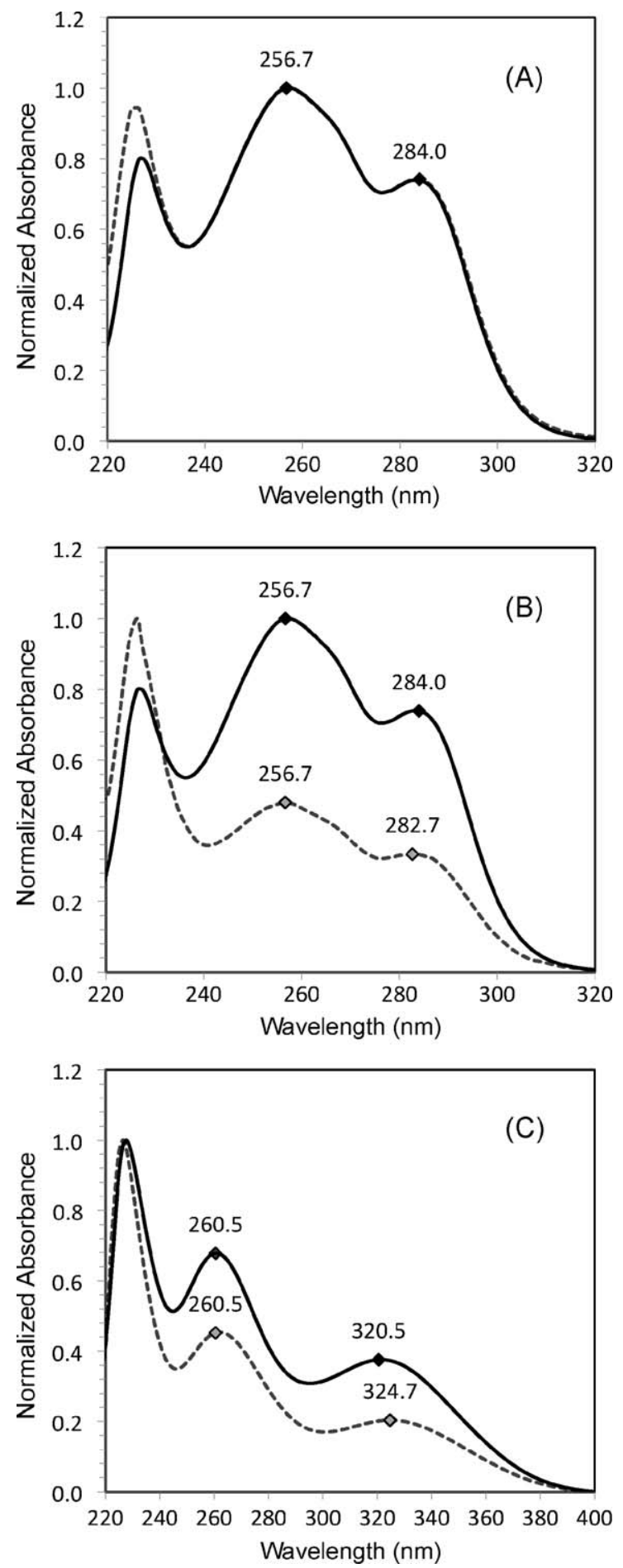

Fig. 5. UV-vis spectra of compounds present in the biotransformation experiments. Comparison of normalized UV-vis spectra of: (A) (-) D-DIBOA analytical standard and (--) compound I from E. coli culture sample identified as D-DIBOA with a 0.9999 correlation coefficient. (B) (-) D-DIBOA analytical standard and (- -) compound II from Nic2 culture sample identified as D-DIBOA with a 0.9342 correlation coefficient. (C) (--) compound III and (-) precursor analytical standard with a 0.9962 correlation coefficient. 
(A) Precursor at $9 \mathrm{~h}$

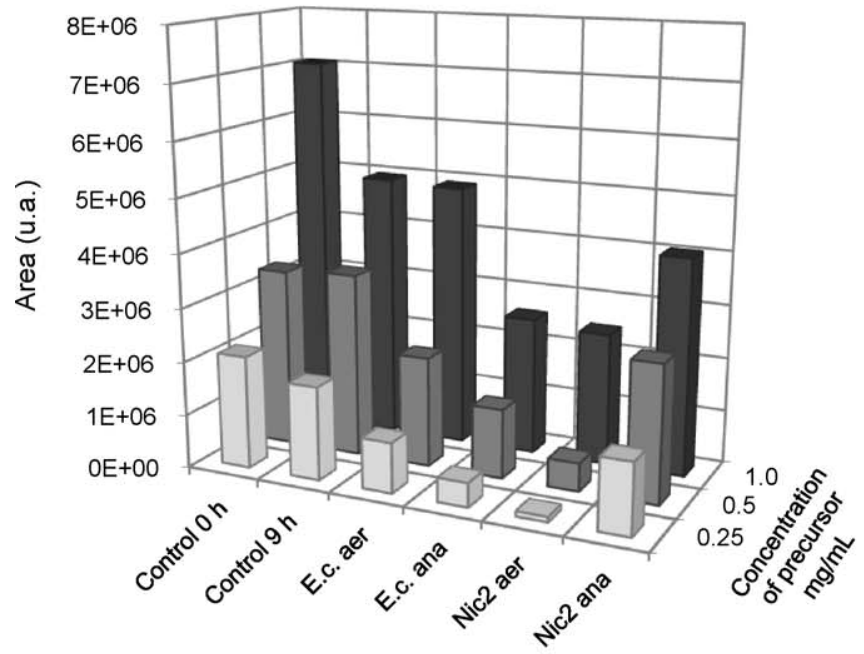

(C) Precursor at $24 \mathrm{~h}$

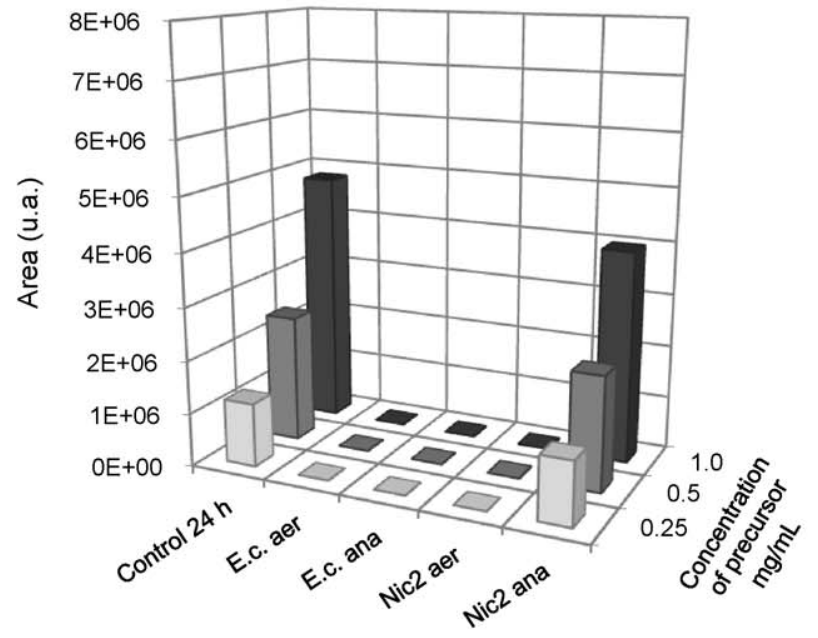

(B) Compounds identified as D-DIBOA at $9 \mathrm{~h}$

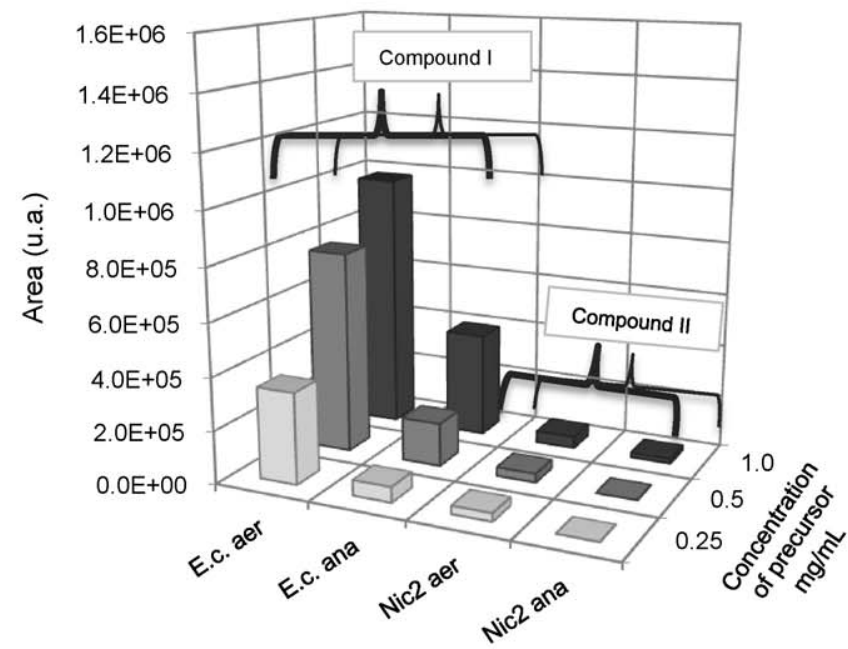

(D) Compounds identified as D-DIBOA at $24 \mathrm{~h}$

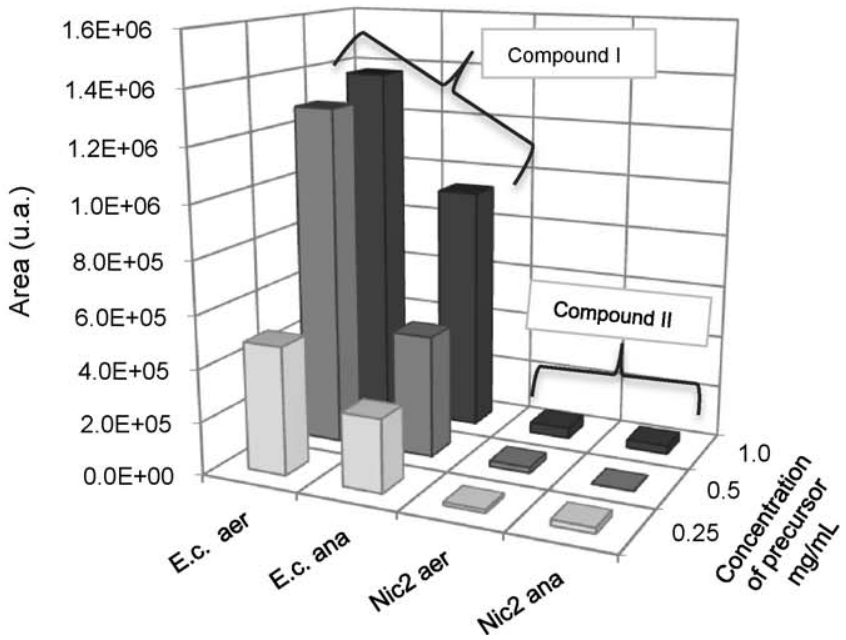

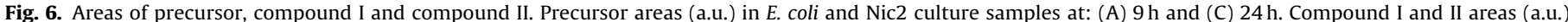

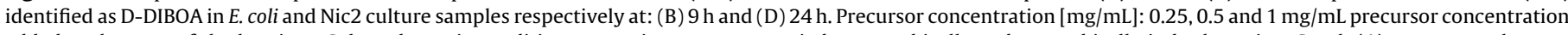

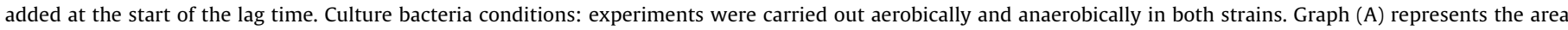
corresponding to abiotic control at 0 and $9 \mathrm{~h}$ to facilitate comparison with the rest of the experiments.

The solvent was removed on a rotary evaporator to give $82.5 \mathrm{mg}$ of extract (AcOEt-A). The extract (AcOEt-A) was separated into four fractions with hexane/AcOEt (6:4) and methanol and the following amounts were obtained: 1 (6.5 mg), 2 (6.3 mg), $3(1.7 \mathrm{mg})$ and 4 $(33.7 \mathrm{mg})$. These fractions were subsequently chromatographed by reverse phase HPLC(C-18 column). In these fractions, the only product identified as D-DIBOA on comparing the RT and UV/vis spectra (Fig. 4(A)) was detected in fraction $4(33.7 \mathrm{mg})$. Furthermore, fraction 4 contained impurities and was therefore purified further on a silica gel column to give $8 \mathrm{mg}$ of pure product.

The ${ }^{1} \mathrm{H}$ NMR (Fig. 7 (B)) and ${ }^{13} \mathrm{C}$ NMR spectra of the pure product in $\mathrm{CDCl}_{3}$ were identical to that of D-DIBOA (Fig. 7(A)) obtained using the procedure described by Macías et al. [6], which unequivocally confirms the structure.

The biotransformation yields obtained for E. coli are shown in Table 1 . The best results were obtained under aerobic conditions in the experiment involving the addition of precursor at $0.5 \mathrm{mg} / \mathrm{mL}$ to give a yield of $20.14 \%$. Under anaerobic conditions the yields were lower - between 8.17 and $9.19 \%$ for the three precursor concentrations tested.
Table 1

Biotransformation yield in E. coli. E.coli culture at $24 \mathrm{~h}$ in aerobic and anaerobic conditions. Precursor added to initial culture. The results are shown as mean \pm Standard Deviation (S.D.).

\begin{tabular}{llc}
\hline Precursor concentration $[\mathrm{mg} / \mathrm{mL}]$ & \multicolumn{2}{l}{ Biotransformation yield \% } \\
\cline { 2 - 3 } & Aerobic & Anaerobic \\
\hline 0.25 & $11.2 \pm 0.8$ & $9.8 \pm 0.8$ \\
0.5 & $20.1 \pm 1.9$ & $8.2 \pm 0.9$ \\
1 & $10.8 \pm 2.2$ & $8.2 \pm 0.6$ \\
\hline
\end{tabular}

\section{Discussion}

In tests with concentrations up to $0.5 \mathrm{mg} / \mathrm{mL}$, the amount of compound I obtained was proportional to the initial concentration of precursor. At higher concentrations a further increase in biotransformation was not observed, a finding that can be attributed to inhibitory effects. Aerobic conditions gave better results in the biotransformation and this could be due in part to the fact that, during these trials, agitation was used and this helps mass transfer, 

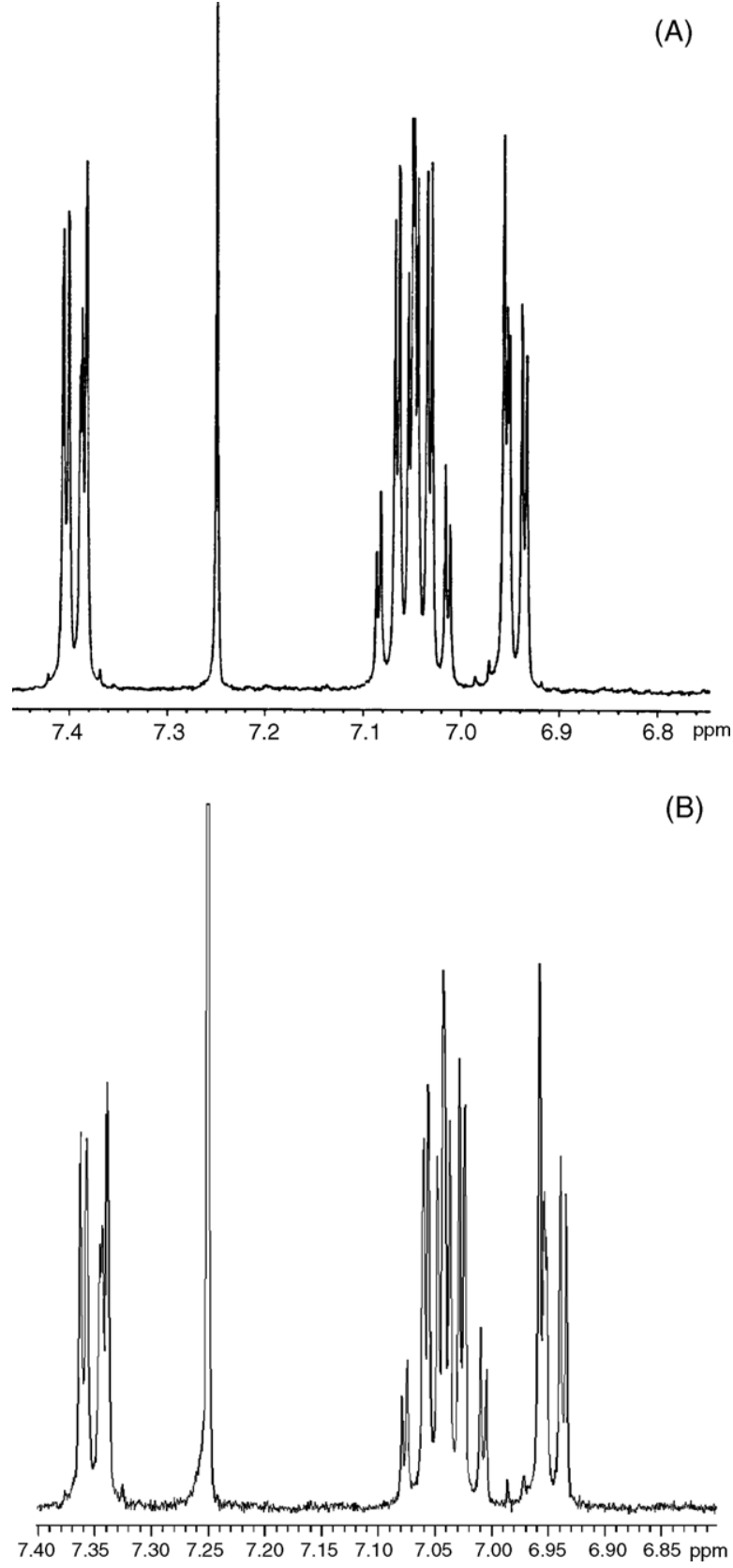

Fig. 7. ${ }^{1} \mathrm{H}$ NMR spectra of D-DIBOA. ${ }^{1} \mathrm{H}$ NMR spectra aromatic ring in ppm (Varian 400 spectrometer) of: (A) D-DIBOA from chemical synthesized and (B) D-DIBOA from E. coli transformation. Both samples were prepared in $\mathrm{CDCl}_{3}$.

thus allowing a better precursor/cell interaction. These difference could be due that bacteria be genetically favoured to carried out this biotransformation in aerobic condition.

The spectroscopic data for compound I from experiments with E. coli are identical to those reported by Atkinson et al. [24] and this compound was identified as D-DIBOA. The transformation of the precursor can be explained in terms of the presence of nitroreductase enzymes, which are involved in the reduction of the nitro group of the precursor.

The biotransformation could be similar to that involved in the reduction of TNT to the corresponding amines [17] as both compounds contain an aromatic ring and a nitro group at C'5. In addition, E. coli is able to reduce these nitroaromatic compounds, most of which are toxic and mutagenic, to the corresponding amines through three types of nitroreductase [25]: two oxygen- insensitive (Type I) and an oxygen-sensitive (Type II) [26] type. In our case, it is possible that type I is involved as the biotransformation occurs equally well in both oxygenic and anoxygenic conditions.

As far as biotransformation yields are concerned, the biological process is still inefficient. These results highlight several variables that could be changed in future studies to achieve better operating performance: e.g. the culture medium composition, the concentration of precursor, the effect of iron concentration, the agitation rate and the purification method.

In conclusion, these results confirm that the strain E. coli used in this study is capable of biotransforming the precursor to DDIBOA, although the yield of this biotransformation is still low. The results also suggest that the enzymes of $E$. coli nitroreductases, as described in the literature, may be present in the strains used and may participate in the reduction of the nitro group of the precursor. The objectives for future work are the identification of the enzymes responsible for the biotransformation using molecular biology techniques and the optimization of operational variables in order to increase the production yields.

\section{Acknowledgments}

This work was supported by the Consejería de Innovación, Ciencia y Empresa de la Junta de Andalucía through the Project for Excellence P06-01399 (2006) The authors wish to thank the Faculty of Biology, Universidad de la Habana (Cuba), who provided the strain $S$. marcescens used in this work.

\section{References}

[1] Duke SO. Naturally occurring chemical compounds as herbicides. Rev Weed Sci 1986;2:15-44

[2] Virtanen AI, Hietala PK. The structures of the precursors of benzoxazolinone in rye plants II. Suom Kemistil B 1959;32:252.

[3] Hamilton RH, Bandurski RS, Reusch WH. Isolation and characterization of a cyclic hydroxamate from Zea mays. Cereal Chem 1962;39:107-13.

[4] Macías FA, Marín D, Oliveros-Bastida A, Molinillo JMG. Rediscovering the bioactivity and ecological role of 1,4-benzoxazinones. Nat Prod Rep (UK) 2009;26:478-89.

[5] Macías FA, Molinillo JM, Galindo JC, Varela RM, Simonet AM, Castellano D. The use of allelopathic studies in the search for natural herbicides. J Crop Prod 2001;4:237-55.

[6] Macías FA, Marín D, Oliveros-Bastidas A, Chinchilla D, Simonet AM, Molinillo JMG. Isolation and synthesis of allelochemicals from Gramineae: benzoxazinones and related compounds. J Agric Food Chem 2006;54:991-1000.

[7] Jernow JL, Rosen P. 2H-1,4-Benzoxazin-3(4H)-ones. U.S. Patent 3,862,180; 1975.

[8] Coutts RT, Hindmarsh KW. 4-Benzoxazine hydroxamic acids and related compounds. Can J Pharm Sci 1966;1:11-7.

[9] Sicker D, Hartenstein $\mathrm{H}$. A new general approach to the 2-hydroxy-2H-1,4benzoxazin-3 $(4 \mathrm{H})$-one skeleton via diisobutylaluminum hydride reduction of 2,3-dioxo-1,4-benzoxazines. Synthesis 1993;8:771-2.

[10] Macías FA, Oliveros-Bastidas A, Marín D, Carrera C, Chinchilla N, Molinillo JMG. Plant biocommunicators: their phytotoxicity, degradation studies and potential use as herbicide models. Phytochem Rev 2008;7:179-94.

[11] Montpas S, Samson J, Langlois E, Lei J, Piché Y, Chênevert R. Degradation of 2,4,6-trinitrotoluene by Serratia marcescens. Biotechnol Lett 1997;19: 291-4.

[12] Yin H, Wood TK, Smets BF. Reductive transformation of TNT by Escherichia coli: pathway description. Appl Microbiol Biotechnol 2005;67:397-404.

[13] Boopathy R, Manning JF. Characterization of partial anaerobic metabolic pathway for 2,4,6-trinitrotoluene degradation by a sulfate-reducing bacterial consortium. Can J Microbiol 1996;42:1203-8.

[14] Ederer MM, Lewis TA, Crawford RL. 2,4,6-Trinitrotoluene (TNT) transformation by clostridia isolated from a munition-fed bioreactor: comparison with nonadapted bacteria. J Ind Microbiol Biotechnol 1997;18:82-8.

[15] Hughes JB, Wang CW, Bhadra R, Richardson A, Bennett GN, Rudolph F. Reduction of 2,4,6-trinitrotoluene by Clostridium acetobutylicum through hydroxylamino intermediates. Environ Toxicol Chem 1998;17:343-8.

[16] Fiorella PD, Spain JC. Transformation of 2,4,6-Trinitrotoluene by Pseudomonas pseudoalcaligenes JS52. Appl Environ Microbiol 1997;63(5):200715.

[17] Esteve-Núñez A, Caballero A, Ramos JL. Biological degradation of 2,4,6trinitrotoluene. Microbiol Mol Biol Rev 2001;65:335-52. 
[18] González-Pérez MM, Van Dillewijn P, Wittich RM, Ramos JL. Escherichia coli has multiple enzymes that attack TNT and release nitrogen for growth. Environ Microbiol 2007;9:1535-40.

[19] Gibson SN, Schroeder M, Steiner W, Gübitz M. Biodegradation of 2,4,6trinitrotoluene (TNT): An enzymatic perspective. Biocatal Biotransform 2005;23:53-69.

[20] Hannink N, Rosser SJ, French CE, Basran A, Murray JAH, Nicklin S, et al. Phytodetoxification of TNT by transgenic plants expressing a bacterial nitroreductase. Nat Biotechnol 2001;19:1168-72.

[21] Williams RE, Rathbone DA, Scrutton NS, Bruce NC. Biotransformation of explosives by the old yellow enzyme family of flavoproteins. Appl Environ Microbiol 2004;70:3566-74.

[22] Marrero J, Corchero D, Peguero M, Batista R, Díaz A, Rodríguez M, et al. Microbial activities in Cuban serpentine soils: Nickel-Cobalt resistant bacteria. In: 11th International Symposium on Microbial Ecology. Vienna (Austria) ISME-11; 2007.

[23] Gamazo C, López-Goñi I, Díaz R. Manual práctico de Microbiología. Barcelona: Masson; 2005. p. 227.

[24] Atkinson J, Morand P, Arnason JT, Niemeyer HM, Bravo HR. Analogues of the cyclic hydroxamic acid 2,4-dihydroxy-7-methoxy-2H-1,4-benzoxazin3-one (DIMBOA): decomposition to benzoxazolinones and reaction with $\beta$-mercaptoethanol. J Org Chem 1991;56:1788-800.

[25] Whiteway JP, Koziarz J, Veall N, Sandhu P, Kumar B, Hoecher, et al. Oxygen-insensitive nitroreductases: analysis of theroles of $n f s A$ and $n s f B$ in development of resistance to 5-nitrofuran derivatives in Escherichia coli. J Bacteriol 1998;180:5529-39.

[26] Marvin-Sikkema FD, de Bont JAM. Degradation of nitroaromatic compounds by microorganisms. Appl Microbiol Biotechnol 1994;42:499-507. 